British Journal of Nutrition (2022), 127, 1352-1357

doi: $10.1017 / \mathrm{S} 0007114521002373$

(C) The Author(s), 2021. Published by Cambridge University Press on behalf of The Nutrition Society. This is an Open Access article, distributed under the terms of the Creative Commons Attribution licence (http://creativecommons.org/licenses/by/4.0/), which permits unrestricted re-use, distribution, and reproduction in any medium, provided the original work is properly cited.

\title{
Lower carbohydrate diets for adults with type 2 diabetes
}

\author{
Mamta Singh $^{1}$, Estella S. Hung ${ }^{1 *}$, Adrienne Cullum ${ }^{1}$, Rachel E. Allen ${ }^{1}$, Peter J. Aggett ${ }^{2}$, Pamela Dyson ${ }^{2}$, \\ Nita G. Forouhi ${ }^{2}$, Darren C. Greenwood ${ }^{2}$, Rachel Pryke ${ }^{2}$, Roy Taylor ${ }^{2}$, Douglas Twenefour $^{2}$, Ruth Waxman ${ }^{2}$ \\ and Ian S. Young ${ }^{2}$ \\ ${ }^{1}$ SACN Secretariat, Department of Health and Social Care, Office for Health Improvement E Disparities, London, \\ United Kingdom \\ ${ }^{2}$ SACN Joint Working Group Member, Department of Health and Social Care, Office for Health Improvement E Disparities, \\ London, United Kingdom
}

(Submitted 20 May 2021 - Final revision received 22 June 2021 - Accepted 23 June 2021 - First published online 1 November 2021)

\section{Background}

In May 2021, the Scientific Advisory Committee on Nutrition (SACN) published a risk assessment on lower carbohydrate diets for adults with type 2 diabetes (T2D $)^{(1)}$. The purpose of the report was to review the evidence on 'low'-carbohydrate diets compared with the current UK government advice on carbohydrate intake for adults with T2D. However, since there is no agreed and widely utilised definition of a 'low'-carbohydrate diet, comparisons in the report were between lower and higher carbohydrate diets. SACN's remit is to assess the risks and benefits of nutrients, dietary patterns, food or food components for health by evaluating scientific evidence and to make dietary recommendations for the UK based on its assessment ${ }^{(2)}$. SACN has a public health focus and only considers evidence in healthy populations unless specifically requested to do otherwise. Since the Committee does not usually make recommendations relating to clinical conditions, a joint working group (WG) was established in 2017 to consider this issue. The WG comprised members of SACN and members nominated by Diabetes UK, the British Dietetic Association, Royal College of Physicians and Royal College of General Practitioners. Representatives from NHS England and NHS Health Improvement, the National Institute for Health and Care Excellence and devolved health departments were also invited to observe the WG. The WG was jointly chaired by SACN and Diabetes UK.

\section{Key words: Lower carbohydrate diets: Type 2 diabetes: Adults: Randomised controlled trials: Scientific Advisory Committee on} Nutrition

The terms of reference for the working group were to review the evidence on lower carbohydrate diets (alongside higher fat and/ or higher protein) compared with the current government advice for adults with type 2 diabetes (T2D); consider the impact, in adults with T2D, of lower compared with higher carbohydrate diets on markers and clinical outcomes of T2D including any potential adverse effects and make recommendations based on the review of the evidence. Its remit did not include consideration of the wider management of $\mathrm{T} 2 \mathrm{D}$, studies of children, people with pre-diabetes, type 1 diabetes or gestational diabetes.

\section{Diabetes}

Diabetes is a condition in which the body does not produce sufficient insulin to regulate blood glucose levels and the insulin produced does not work effectively. This leads to elevated blood glucose concentrations (hyperglycaemia) which causes damage to blood vessels and nerves.
There are two main types of diabetes: type 1 diabetes and T2D. There are also other forms such as gestational diabetes and rare genetic forms such as maturity onset diabetes of the young. In 2018, an estimated 4.7 million people in the UK had diabetes ${ }^{(4)}$. This included about 3.8 million people with diagnosed diabetes and an estimated 1 million people who were undiagnosed.

T2D accounts for about $90 \%$ of all cases of diabetes in the $\mathrm{UK}^{(4-6)}$ and occurs as a result of reduced beta-cell insulin secretion and increased insulin resistance ${ }^{(7)}$. Although several non-modifiable risk factors such as age, family history and ethnicity are associated with increased T2D risk, about 80-85\% of an individual's risk of developing T2D is associated with obesity ${ }^{(8)}$, a modifiable risk factor.

\section{Current UK government advice}

Current UK government advice on carbohydrate intake is based on recommendations made by Scientific Advisory Committee on

Abbreviations: MA, meta-analyses; RCT, randomised controlled trial; SACN, Scientific Advisory Committee on Nutrition; SR, systematic review; T2D, type 2 diabetes.

* Corresponding author: Estella S. Hung, email estella.hung@dhsc.gov.uk

This article has been co-published with permission in British Journal of Nutrition and Diabetic Medicine. 
Nutrition (SACN) following its review on carbohydrates and health ${ }^{(3)}$. The evidence considered in the SACN review on carbohydrates comprised studies in the general population, and recommendations were for the UK general population.

Current UK government advice for the general population is that approximately $50 \%$ of total dietary energy should be obtained from carbohydrates, mainly from starchy foods consisting of high fibre or whole-grain food where possible. It is recommended that average population intake of free sugars should not exceed $5 \%$ of total dietary energy and that adults should achieve a daily dietary fibre intake of $30 \mathrm{~g} / \mathrm{d}$.

There are no separate recommendations on carbohydrate intake for adults with T2D, and the advice for the general UK population thus applies to those with T2D.

In England, the National Institute for Health and Care Excellence has issued guidelines for the identification, diagnosis and management of T2D including dietary advice that is in line with UK government advice for the general population ${ }^{(9)}$. The Scottish Intercollegiate Guidelines Network has also issued guidelines on management of diabetes including offering adults with T2D a range of dietary options for achieving weight loss that may also improve glycaemic control ${ }^{(10)}$.

The ultimate aim of T2D management and treatment is to reduce and maintain glycated haemoglobin (HbA1c) concentration at a value below the cut-off for the definition of T2D. However, any reduction in HbA1c reflects an improvement in the degree of T2D control. Improvement of blood lipids and blood pressure is also an important treatment goal.

Management of T2D usually involves behavioural interventions (including diet, physical activity, smoking cessation and reduced alcohol intake) and/or medications. Treatment may also include bariatric surgery to reduce weight.

A reduction in energy intake is an important part of the behavioural interventions recommended to people with T2D who are living with overweight or obesity. The aim of reducing energy intake is weight loss, which in turn improves glycaemic control. Glycaemic index and glycaemic load are measures of the post-prandial blood glucose response to foods.

Both Diabetes UK and the American Diabetes Association emphasise tailoring advice to the individual, and both note the lack of clear evidence for a specific dietary intake of carbohydrate for those with $\mathrm{T} 2 \mathrm{D}^{(11,12)}$.

\section{Purpose of review}

SACN initiated this evidence review in response to a request from Public Health England, for a systematic assessment of the scientific evidence on 'low'-carbohydrate diets, in recognition that such diets are gaining attention and increasingly being promoted.

\section{Methods}

SACN's Framework for the Evaluation of Evidence ${ }^{(2)}$ was used as the basis for assessing the evidence. Public Health England conducted a search of key online databases: MEDLINE, Embase, the Cochrane Library (CDSR and DARE), National Institute for Health and Care Excellence evidence, TRIP and Google
Table 1. Categories of dietary carbohydrate intakes based on Feinman et al. $(2015)^{(13)}$ and Accurso et al. (2008) $)^{(14)}$

\begin{tabular}{lcc}
\hline & \multicolumn{2}{c}{ Amount of carbohydrate } \\
\cline { 2 - 3 } Description & $\mathrm{g} / \mathrm{d}$ & $\%$ TE (based on $2000 \mathrm{kcal} / \mathrm{d}$ ) \\
\hline Very low carbohydrate ${ }^{\dagger}$ & $20-50$ & $\leq 10$ \\
Low carbohydrate & $>50-<130$ & $>10-<26$ \\
Moderate carbohydrate & $130-230$ & $26-45$ \\
High carbohydrate & $>230$ & $>45$ \\
\hline
\end{tabular}

$\dagger$ Also referred to as ketogenic diets.

Scholar to identify relevant English language articles published between 1980 and 30 September 2018, using an agreed list of search terms focusing on lower carbohydrate diets and T2D. There was a particular interest in the following primary outcomes: weight change ( $\geq 12$ months), glycated $\mathrm{Hb}$ (HbA1c), a marker of impaired glycaemic control ( $\geq 3$ months), and secondary outcomes: body weight ( $\geq 3$ to $<12$ months), fasting plasma glucose ( $\geq 3$ months), blood lipid profiles ( $\geq 3$ months) and medication use

SACN invited interested parties to highlight relevant evidence which satisfied the inclusion criteria for the report through a call for evidence from 9 February to 7 March 2018.

The report included evidence from systematic reviews (SR) and meta-analyses (MA) and pooled analyses of randomised controlled trials (RCT) and prospective cohort studies comparing the impact of lower $v$. higher carbohydrate diets on markers and clinical outcomes of T2D. It also included RCT published after the most recent SR, MA and pooled analyses of RCT ${ }^{(2)}$. Only SR that included studies with a minimum duration of 3 months and individuals with pre-diagnosed T2D (as defined in the primary RCT) when they entered the study were considered. Two reviewers independently screened titles and abstracts of the publications identified by the literature search and subsequently assessed full-text articles. Any differences were resolved by consensus.

For the purposes of this report, in order to enable comparisons of carbohydrate intake across the studies under consideration, the classification proposed by Feinman et al. $(2015)^{(13)}$ was adopted as the basis for categorisation of carbohydrate intake (See Table 1).

The quality of included SR and MA was assessed by the approach outlined in the SACN Framework for the Evaluation of Evidence ${ }^{(2)}$ and the AMSTAR 2 tool $^{(15)}$. Expert judgement, based on pre-specified criteria, was used to grade the strength of the evidence (adequate, moderate, limited, inconsistent or insufficient) for the primary and secondary outcomes. Only outcomes where the evidence base was graded as adequate or moderate were used to inform recommendations.

\section{Evidence identified}

After removal of duplicates, the online database search identified 3169 abstracts which were screened for eligibility. Full texts of nineteen potentially relevant SR with MA were retrieved and assessed. Out of these, fifteen were excluded. Five additional publications that met the inclusion criteria were identified by SACN working group members. In total, nine publications met 


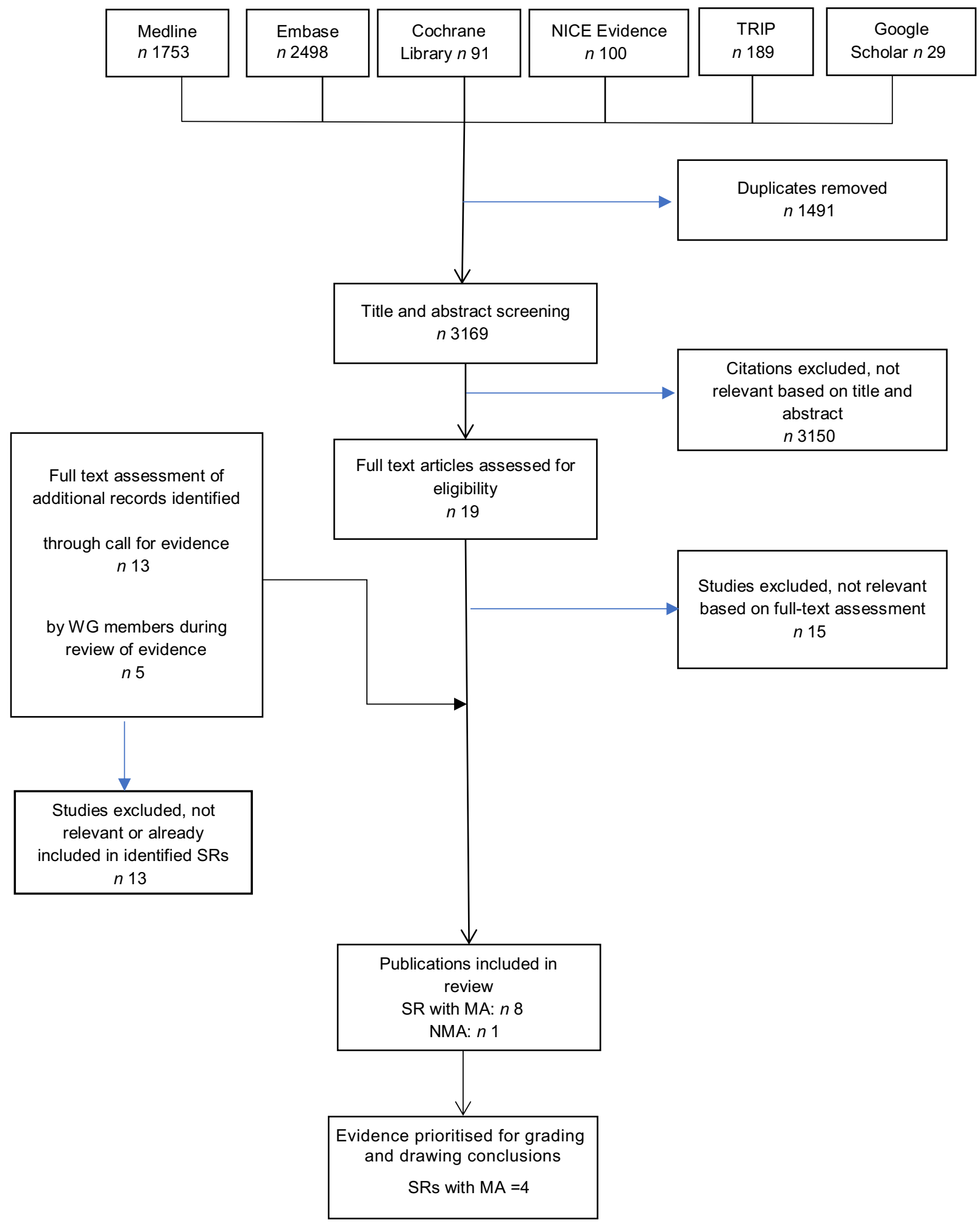

Fig. 1. Flow diagram showing the number of publications assessed for eligibility and included in the evidence review.

the criteria and were included for data extraction, including eight SR with MA and one network meta-analysis (See Fig. 1).

After detailed assessment, results from four SR with $\mathrm{MA}^{(16-19)}$ were used to grade the evidence and draw conclusions. This is because they were more recent, had larger numbers of participants and were considered to be of better quality based on the SACN Framework for the Evaluation of Evidence ${ }^{(2)}$ and AMSTAR 2 criteria ${ }^{(15)}$. Out of the thirty-six publications included in the MA, eighteen reported outcomes in the shorter term (minimum duration of 3 months) only and eighteen reported 
outcomes in the longer term (minimum duration of 12 months). In this report, shorter term refers to studies or assessments that reported at $\geq 3$ to 6 months (since all except 1 of the time points included in shorter-term studies or assessments did not extend beyond 6 months) and longer term refers to studies that reported outcomes at $\geq 12$ months.

Body weight. The evidence for lower compared with higher carbohydrate diets on body weight was inconsistent in the shorter term ( $\geq 3$ to 6 months); there was a greater reduction in body weight with lower compared with higher carbohydrate diets at 3 months, but this difference was not observed between 3 and 6 months or at 6 months. There was adequate evidence for no difference in effect between lower and higher carbohydrate diets in reducing body weight in the longer term ( $\geq 12$ months).

$H b A 1 c$. There was adequate evidence of a greater reduction in HbA1c with lower compared with higher carbohydrate diets in the shorter term ( $\geq 3$ to 6 months). The evidence was inconsistent in longer-term studies with a duration of 12 up to 24 months. There was adequate evidence for no difference between lower and higher carbohydrate diets on HbA1c change in longer-term studies at 24 months.

Fasting plasma glucose. There was moderate evidence of a greater reduction in fasting plasma glucose with lower compared with the higher carbohydrate diets in the shorter term $(\geq 3$ to 6 months). There was insufficient evidence to assess if there was a difference between lower and higher carbohydrate diets on fasting plasma glucose in the longer term ( $\geq 12$ months).

Serum total cholesterol. There was moderate evidence for no difference in effect between lower and higher carbohydrate diets in reducing serum total cholesterol in the shorter term $(\geq 3$ to 6 months). There was adequate evidence for no difference in effect between lower and higher carbohydrate diets in reducing serum total cholesterol in the longer term ( $\geq 12$ months).

Serum TAG. There was adequate evidence of a greater reduction in serum TAG with lower compared with higher carbohydrate diets in the shorter term ( $\geq 3$ to 6 months). The evidence was inconsistent in the longer term ( $\geq 12$ months).

Serum LDL-cholesterol. There was adequate evidence for no difference in effect between lower and higher carbohydrate diets on change in serum LDL-cholesterol in the shorter term $(\geq 3$ to 6 months) and in the longer term ( $\geq 12$ months).

Serum HDL-cholesterol. The evidence on lower compared with higher carbohydrate diets on serum HDL-cholesterol was inconsistent in the shorter term ( $\geq 3$ to 6 months) and longer term ( $\geq 12$ months)

Changes in medication use. There was moderate evidence of a greater reduction in medication use with lower compared with higher carbohydrate diets. This outcome was not assessed according to study duration. It was not possible to assess consistency in effect size.

\section{Limitations of the evidence base}

The overall quality of the evidence base was limited by a number of uncertainties in the data, including: variability in the definition of a low-carbohydrate diet; smaller than prescribed differences in reported carbohydrate intakes between lower and higher carbohydrate diets; inherent inaccuracies in estimates of selfreported dietary intakes and lack of information on adherence to prescribed diets.

An important limitation was that risk of bias was high or unclear in most of the primary RCT that were included in the MA. This reduces the confidence that can be placed on the estimates of the effects of lower carbohydrate diets on the markers of T2D and clinical outcomes under consideration.

Another important limitation in the evidence base was that shorter-term studies did not assess outcomes beyond 6 months and few longer-term studies assessed outcomes beyond 12 months.

The majority of participants in the primary RCT were living with overweight (BMI $\geq 25$ to $<30 \mathrm{~kg} / \mathrm{m}^{2}$ ) or obesity $\left(\mathrm{BMI} \geq 30 \mathrm{~kg} / \mathrm{m}^{2}\right.$ ). It is not known if reported effects can be generalised to adults with a healthy weight $(\mathrm{BMI} \geq 18.5$ to $<25$ $\mathrm{kg} / \mathrm{m}^{2}$ ).

It is not known if the reported effects of lower carbohydrate diets apply to individuals of different ethnicities since the majority of primary RCT did not report ethnicity of participants and most were conducted in populations that were predominantly White. In those that reported ethnicity, none conducted subgroup analyses based on ethnicity.

This report did not assess evidence on the effect of lower carbohydrate diets in the general population without T2D. It is not known if the reported effects of lower carbohydrate diets in adults with T2D apply to the general adult population without T2D.

Several additional gaps were identified in the evidence base:

- no trials provided information about the type of carbohydrate consumed (e.g. wholegrain, refined grain, free sugars, fibre) or considered how this could affect the outcomes of interest;

- the potential impact of increasing the proportions of other macronutrients (usually fats and/or proteins) to compensate for reduced carbohydrate intake in the lower carbohydrate groups, or the type of macronutrient (e.g. saturated or unsaturated fats; plant- or animal-based proteins), on markers and clinical outcomes of T2D was generally not considered; and

- no trials considered clinical endpoints such as diabetes complications, CVD events or mortality

\section{Conclusions}

Overall, the evidence suggests beneficial effects of lower carbohydrate diets for some outcomes (HbA1c, fasting plasma glucose, serum TAG) in the shorter term (up to 6 months). Since the shorter-term assessments did not report outcomes between 6 and 12 months, it is uncertain if the suggested benefits are maintained beyond 6 months.

Although there was no consistent evidence of reductions in body weight with lower carbohydrate diets, it is not possible, 
from the evidence considered, to separate the effects of weight change from effects of change in carbohydrate intake.

Lower carbohydrate diets may allow reductions in diabetes medication, but interpretation is complicated by inconsistencies in reporting and measurement of changes in medication use.

No differences were observed between higher and lower carbohydrate diets on serum total or LDL-cholesterol either in the shorter ( $\geq 3$ to 6 months) or longer term ( $\geq 12$ months). Evidence on HDL-cholesterol was inconsistent in the shorter ( $\geq 3$ to 6 months) and longer term ( $\geq 12$ months).

In general, there was no difference in adverse events between lower and higher carbohydrate diets but study duration did not extend beyond 12 months in the majority of primary RCT.

\section{Recommendations}

The report states that recommendations are applicable to adults living with T2D and overweight or obesity. There was insufficient evidence to make recommendations for adults living with T2D without overweight or obesity. The report did not assess evidence on the effect of lower carbohydrate diets in the general population without T2D.

The report makes the following recommendations:

- For adults living with T2D and overweight or obesity, a lower carbohydrate diet can be recommended by clinicians as an effective short-term option (up to 6 months) for improving glycaemic control and serum TAG concentrations.

- Individuals living with T2D and overweight or obesity who choose a lower carbohydrate diet should include whole-grain or higher fibre foods, a variety of fruits and vegetables and limit intakes of saturated fats, reflecting current dietary advice for the general population.

- Since the majority of individuals living with T2D have overweight or obesity, weight management remains the primary goal for improving glycaemic control and reducing CVD risk. Health professionals should support any evidence-based dietary approach that helps individuals with T2D to achieve long-term weight reduction.

- Adults living with T2D and overweight or obesity who change to a lower carbohydrate diet and are taking diabetes medication may be at risk of hypoglycaemia. It is recommended that they receive advice and support from their health care team to manage this risk and to make adjustments to their medication as required.

\section{Acknowledgements}

The report was developed by the SACN working group and reviewed and approved by the full SACN committee. A list of working group and SACN members is available here: https://www.gov. uk/government/groups/scientific-advisory-committee-on-nutrition. SACN is an independent committee of Public Health England. The report was funded by Public Health England.

We would also like to thank Professor Ann Prentice from the Medical Research Council (MRC) Nutrition and Bone Health Research Group, Cambridge, who was SACN Chair during the development of the report until May 2020. We would also like to thank Professor Ian Macdonald from the University of Nottingham, who jointly chaired the SACN working group until March 2020.

R. E. A. prepared the first draft of the manuscript, which was revised by A. C. All authors commented on the draft manuscript and approved the final manuscript. R. T. declared receiving lecture fees from pharmaceutical firms Lilly; Jansen; Novartis and Nestle; consultancy fees from Wilmington Healthcare and Lilly; and is author of the book 'Life Without Diabetes'. R. P. declared presenting webinars funded by Novo Nordisk. P. D. is affiliated to OCDEM, Oxford University NHS Foundation Trust, Oxford, UK and NIHR Biomedical Research Centre, Oxford, UK. N. G. F. is affiliated to MRC Epidemiology Unit, University of Cambridge School of Clinical Medicine. She acknowledges support from MRC Epidemiology Unit (MC_UU_00006/3).

Details of declarations of interest for working group and SACN members are available here: https://www.gov.uk/government/ groups/scientific-advisory-committee-on-nutrition\# register-ofinterests

\section{References}

1. Scientific Advisory Committee on Nutrition (SACN) (2021) Lower Carbohydrate Diets for Adults with Type 2 Diabetes. https://www.gov.uk/government/publications/sacn-reportlower-carbohydrate-diets-for-type-2-diabetes (accessed July 2021).

2. Scientific Advisory Committee on Nutrition (SACN) (2020) SACN Framework for the Evaluation of Evidence. https://app.box. com/s/ecdi8ufpleqlzk0pd7odm74m8do2riw9 (accessed April 2021).

3. Scientific Advisory Committee on Nutrition (SACN) (2015) Carbohydrates and Health Report. https://www.gov.uk/ government/publications/sacn-carbohydrates-and-health-report (accessed April 2021).

4. Diabetes UK (2019) Us, Diabetes and a Lot of Facts and Stats. https://www.diabetes.org.uk/resources-s3/2019-11/ facts-stats-update-oct-2019.pdf (accessed September 2021).

5. NHS Digital (2018) National Diabetes Audit - Report 1 Care Processes and Treatment Targets 2017-18. https://digital.nhs. uk/data-and-information/publications/statistical/nationaldiabetes-audit/report-1-care-processes-and-treatment-targets2017-18-short-report (accessed September 2021).

6. NHS Scotland (2020) Scottish Diabetes Survey 2019. https:// www.diabetesinscotland.org.uk/wp-content/uploads/2020/10/ Diabetes-Scottish-Diabetes-Survey-2019.pdf (accessed September 2021.

7. ADA (2019) Classification and diagnosis of diabetes: standards of medical care in diabetes - 2019. Diabetes Care 42, S13-S28.

8. Hauner H (2010) Obesity and Diabetes. Textbook of Diabetes, 4th ed. Oxford: Wiley-Blackwell.

9. NICE (2020) Type 2 Diabetes in Adults: Management [NG28]. https://www.nice.org.uk/guidance/ng28 (accessed June 2021).

10. SIGN (2019) Management of Diabetes. https://www.sign.ac. uk/our-guidelines/pharmacological-management-of-glycaemiccontrol-in-people-with-type-2-diabetes/ (accessed June 2021).

11. Diabetes UK (2021) Position statement for healthcare professionals-remission in adults with Type 2 diabetes. https://www.diabetes.org.uk/professionals/position-statementsreports/statement-remission-type2 (accessed September 2021). 
12. ADA (2020) Facilitating behaviour change and well-being to improve health outcomes: Standards of Medical Care in Diabetes 2020. Diabetes Care 43, S48-S65.

13. Feinman RD, Pogozelski WK, Astrup A, et al. (2015) Dietary carbohydrate restriction as the first approach in diabetes management: critical review and evidence base. Nutrition 31, 1-13.

14. Accurso A, Bernstein RK, Dahlqvist A, et al. (2008) Dietary carbohydrate restriction in type 2 diabetes mellitus and metabolic syndrome: time for a critical appraisal. Nutr Metab 5, 9 .

15. AMSTAR (2017) AMSTAR 2. https://amstar.ca/Amstar-2.php (accessed June 2021).

16. Huntriss R, Campbell M \& Bedwell C (2018) The interpretation and effect of a low-carbohydrate diet in the management of type 2 diabetes: a systematic review and meta-analysis of randomised controlled trials. Eur J Clin Nutr 72, 311-325.

17. Korsmo-Haugen H-K, Brurberg KG, Mann J, et al. (2018) Carbohydrate quantity in the dietary management of type 2 diabetes: a systematic review and meta-analysis. Diabetes Obes Metab 21, 15-27.

18. Sainsbury E, Kizirian NV, Partridge SR, et al. (2018) Effect of dietary carbohydrate restriction on glycemic control in adults with diabetes: a systematic review and meta-analysis. Diabetes Res Clin Pract 139, 239-252.

19. van Zuuren EJ, Fedorowicz Z, Kuijpers T, et al. (2018) Effects of low-carbohydrate-compared with low-fat-diet interventions on metabolic control in people with type 2 diabetes: a systematic review including GRADE assessments. Am J Clin Nutr 108, 300-331. 